\title{
STRUCTURAL AND FUNCTIONAL STUDIES OF PROLINE CATABOLIC ENZYMES AND HUMAN ALDEHYDE DEHYDROGENASES
}

\author{
Min Luo \\ Dr. John J. Tanner, Dissertation Advisor
}

\begin{abstract}
Oxidation of amino acids, like proline catabolism, is a central part of energy metabolism. Proline is oxidized to glutamate by two enzymes: proline dehydrogenase (PRODH) and 1-pyrroline-5-carboxylate dehydrogenase (P5CDH). PRODH catalyzes the first reaction of proline to 1-pyrroline-5-carboxylate (P5C). P5C undergoes a non-enzymatic hydrolysis to glutamate semialdehyde (GSA), which is oxidized to glutamate by a NAD ${ }^{+}$dependent enzyme P5CDH. PRODH and P5CDH are mono-functional enzymes in eukaryotes and Gram-positive bacteria; while in Gram-negative bacteria, the two enzymes are fused into one protein as two domains, known as proline utilization A (PutA). This dissertation work involved structural and functional studies of PRODH, P5CDH, PutA, and human aldehyde dehydrogenases (ALDHs). The results illuminated the substrate recognition for mono-functional PRODH and hot spot oligomerization mechanism for mono-functional $\mathrm{P} 5 \mathrm{CDH}$, also, demonstrated that diethylaminobenzaldehyde (DEAB) is a mechanism based inactivator for aldehyde dehydrogenase 7A1. Furthermore, the C-terminal domain found in PutAs, the only domain without any structural and functional information has been structurally and biochemically characterized.
\end{abstract}

